\title{
Prediction and explanation in the multiverse
}

\author{
J. Garriga ${ }^{1}$ and A. Vilenkin ${ }^{2}$ \\ ${ }^{1}$ Departament de Física Fonamental, Universitat de Barcelona, Martí i Franquès 1, 08193 Barcelona, Spain \\ ${ }^{2}$ Institute of Cosmology, Department of Physics and Astronomy, Tufts University, Medford, Massachusetts 02155, USA
}

(Received 22 November 2007; published 26 February 2008)

Probabilities in the multiverse can be calculated by assuming that we are typical representatives in a given reference class. But is this class well defined? What should be included in the ensemble in which we are supposed to be typical? There is a widespread belief that this question is inherently vague, and that there are various possible choices for the types of reference objects which should be counted in. Here we argue that the "ideal" reference class (for the purpose of making predictions) can be defined unambiguously in a rather precise way, as the set of all observers with identical information content. When the observers in a given class perform an experiment, the class branches into subclasses who learn different information from the outcome of that experiment. The probabilities for the different outcomes are defined as the relative numbers of observers in each subclass. For practical purposes, wider reference classes can be used, where we trace over all information which is uncorrelated to the outcome of the experiment, or whose correlation with it is beyond our current understanding. We argue that, once we have gathered all practically available evidence, the optimal strategy for making predictions is to consider ourselves typical in any reference class we belong to, unless we have evidence to the contrary. In the latter case, the class must be correspondingly narrowed.

DOI: 10.1103/PhysRevD.77.043526

PACS numbers: $98.80 . \mathrm{Cq}$

\section{INTRODUCTION}

The task of assigning probabilities to the outcomes of observations has a long tradition in physics, and we are used to the fact that such predictions improve as we progress in our understanding of the fundamental theory. Yet, the current situation in cosmology is paradoxical. The inflationary scenario, which is now the leading cosmological paradigm, suggests a very large universe with a variety of different environments. For example, the density fluctuations in this scenario are stochastic variables determined by quantum fluctuations of the inflaton field. The fluctuation amplitudes and spectra are different in different parts of the universe. In axion models of dark matter, the dark matter density is also a random variable, determined by the local amplitude of the axion field. In this situation, it seems pertinent to ask the following question. Given some information about the properties of our own environment, can we assign probabilities to any of the remaining properties? Despite its simplicity, there is no consensus on how this question should be tackled, or whether it has an answer at all.

The problem is particularly acute when the fundamental theory admits a multitude of solutions describing vacua with different values of the low-energy constants of nature, as may be the case in string theory [1]. In the cosmological context, high-energy vacua drive an exponential inflationary expansion of the universe. Transitions between different vacua can occur through tunneling and quantum diffusion, so "pocket universes" filled with all possible kinds of vacua are constantly being formed and the entire landscape of vacua can be explored [2]. In this scenario, the local values of the constants cannot be predicted with certainty, but we may be able to make statistical predictions. The prediction of the observed value of the cosmological constant [3-6] is a notable success of this approach, but the methods used for that prediction have been a subject of much recent criticism [7-9].

In anthropic predictions of this sort one usually assumes that we are typical representatives in some reference class of observers, and the arbitrariness in the choice of the reference class is the main target of the criticism. Should the reference class include all living intelligent creatures? If so, how do we define life and intelligence? Should chimpanzees be included? And whatever our choice of reference class, how do we know that we are typical in that class?

This situation is to be contrasted with that in quantum mechanics, where we imagine an infinite ensemble of identical experiments performed by identical observers. (The observers are actually existing in the many worlds interpretation.) Once the experiment is performed, the ensemble branches into a number of subensembles corresponding to the different outcomes of the experiment, and the probabilities are identified with the corresponding branching ratios.

This paper is an attempt to clarify the definitions of probability and reference class in multiverse models. We shall argue that, despite a widespread belief to the contrary, these concepts can be defined unambiguously in a rather precise way (which is somewhat analogous to the one based on the branching ratios in quantum mechanics).

We should mention that there is a further complication in the case of the inflationary multiverse. The problem is that the number of elements in any given reference class is 
infinite, and the relative number of elements in each subclass depends on how we regulate the infinities. This is known as the measure problem, which is currently a subject of active research (for recent discussions, see [10-15]). Here we shall not discuss this problem any further, since we are addressing a different set of issues which are relevant to the results obtained from any given regulator.

\section{THE BRANCHING REFERENCE CLASS}

For any given event, eternal inflation leads to an infinite number of occurrences, coexisting in different regions of a single spacetime continuum. In the inflationary "multiverse" there are an infinite number of pocket universes of any given type. And if an event can occur inside of a given pocket, then this pocket will contain not just one but an infinite number of such events.

Suppose a measurement $\mathcal{M}$ is about to be made, which can yield a number of possible data sets $D_{j}$, and some "observer" (not necessarily a member of the observational team) wants to predict the outcome of this measurement. We can characterize the observer by the information she has that is relevant for the experiment, as well as by all the irrelevant information, such as her address, the name of her dog, etc. In other words, the observer is characterized by the full information content at her disposal. The key point is that there is an infinite number of observers with identical information content in the multiverse [16]. We suggest that, ideally, this set of observers should be chosen as the reference class $C$, to be used for making predictions. (A much wider class is usually used for practical considerations; see below.)

Once the measurement is made, observers get new information from the data, and the class $C$ splits into a number of subclasses $C_{j}$ corresponding to different outcomes $D_{j}$. The observer information content is the same within each subclass, but differs from one subclass to another. The probability of a data set $D_{j}$ should be identified with the fraction $f_{j}$ of observers who end up in subclass $C_{j}$,

$$
P\left(D_{j}\right)=f_{j}
$$

This equation assigns equal statistical weight to all observers in class $C$. In other words, we think of ourselves as being randomly picked from that class of observers. ${ }^{1}$ This is justified by the fact that all observers in $C$ have identical

\footnotetext{
${ }^{1}$ The proposal of using the full information content of observers for making predictions has also been advanced by Neal [17]. However, he assumed a finite universe, small enough, so that observers can be uniquely specified by their information content. This is very different from the situation we are discussing here.
}

information content and thus have no way of distinguishing between one another. ${ }^{2}$

If another measurement $\mathcal{M}^{\prime}$ is made after $\mathcal{M}$, the probabilities for its possible outcomes $D^{\prime}{ }_{j}$ should be updated using the Bayes formula,

$$
P\left(D_{j}^{\prime} \mid D_{i}\right)=\frac{P\left(D_{j}^{\prime}, D_{i}\right)}{P\left(D_{i}\right)}
$$

The probabilities for $D^{\prime}{ }_{j}$ assigned by observers in different subclasses $C_{j}$ are, of course, generally different. Every subsequent measurement will lead to a further splitting of reference classes. The resulting "branching tree" of reference classes is similar to the branching wave function in the many worlds interpretation of quantum mechanics.

It should be stressed that the above discussion assumes that we have a theory allowing us to calculate the probabilities $P\left(D_{j}\right)$. Agreement (or lack thereof) of these probabilities with the data can be used to evaluate the theory, as we shall discuss below, in Sec. V

\section{THE PRINCIPLE OF MEDIOCRITY}

Using the full information content of observers for calculation of probabilities is not a very practical proposition. One only needs to use the relevant information which has non-negligible correlation with the data. Moreover, our models of the multiverse are too crude and our understanding of life and intelligence too rudimentary to account for more than very basic information. Thus, by necessity, we need to consider a reference class of observers specified by a small subset of all available information, and thus much wider than the class $C$ defined above.

Suppose, for example, we want to predict the sum of the three neutrino masses, $m_{\nu}$. (The mass differences are already known from neutrino oscillation measurements.) For this purpose, we could consider the reference class $C^{(\nu)}$ of all humanlike observers who measured the same values for all constants of nature other than $m_{\nu}$. The probability distribution $P\left(m_{\nu}\right)$ can then be calculated [18], with some additional assumptions about the landscape of possible vacua. This distribution gives the probability that an observer randomly picked in the class $C^{(\nu)}$ will measure a given value of $m_{\nu}$. Serious doubts have been raised, however, that such distributions can be used for making predictions in our local region [7-9].

\footnotetext{
${ }^{2}$ The actual calculation of the probabilities is complicated by the fact that the number $N$ of observers in $C$ is infinite. In order to calculate the fractions $f_{j}$, one has to impose some sort of a cutoff on $N$ and then remove the cutoff at the end of the calculation. The result, however, turns out to be sensitive to the cutoff procedure. This is the so-called measure problem which we referred to at the end of the introduction. Since our considerations apply to the results obtained from any cutoff procedure, we shall not dwell on this problem any further.
} 
The class $C^{(\nu)}$ is specified by very limited information, which does not include the amplitudes of the cosmic microwave background (CMB) multipoles, observer's addresses, etc. Much of the omitted information is irrelevant, that is, has negligible correlation with the value of $m_{\nu}$. But some information may be relevant (e.g., the abundance of galaxy clusters is known to be correlated with $m_{\nu}$ ), and for some other information the relevance may be hard to assess. Why, then, should we think of ourselves as typical (randomly selected) in the reference class $C^{(\nu)}$ ? This is what Hartle and Srednicki [7] call "the selection fallacy."

Hartle and Srednicki have argued that we should never assume ourselves to be typical representatives of some reference class of observers, unless we have evidence to back up that assumption. We disagree. ${ }^{3}$ We would suggest that, on the contrary, we should assume ourselves to be typical in any class that we belong to, unless there is some evidence to the contrary. This is a statement of the principle of mediocrity. ${ }^{4}$

Even though observers in class $C^{(\nu)}$ may suspect that some relevant information may be missing, the principle of mediocrity recommends that they should make their predictions using the probability distribution for that class. When the missing information becomes available, the class $C^{(\nu)}$ will split into subclasses $C^{(\nu) \prime}, C^{(\nu) \prime \prime}$, etc. Observers in $C^{(\nu)}$ would be able to make more accurate predictions if they could calculate the distributions for their respective subclasses. But in the absence of such distributions, they should base their bets on the distribution for $C^{(\nu)}$. Some of them will guess incorrectly, but there will be more winners than losers. This is the justification for the principle of mediocrity: it simply improves the odds.

The principle of mediocrity is widely used in physics, although it is rarely acknowledged explicitly. For example, an experimentalist representing the result of a measurement as $x \pm \delta x$ thinks of his data as a random sample drawn from a Gaussian distribution of width $\delta x$. Similarly, cosmologists think of the CMB multipoles as randomly drawn from Gaussian distributions. Without assuming that the data are typical in this sense, we would have to conclude that the measurement gives us no information about the quantity being measured.

\footnotetext{
${ }^{3}$ See also the article by Page [19] for a critique of Hartle and Srednicki.

${ }^{4}$ The original formulation of the principle of mediocrity in [4] asserts that we should regard our civilization as randomly picked among all civilizations in the multiverse. Similar ideas have been earlier discussed by Gott [20] and Leslie [21] and about the same time by Page [22]. Our formulation here is more general. A related proposal is the "self-sampling assumption" introduced by Bostrom [23]: "One should reason as if one were a random sample from the set of all observers in one's reference class." The problem with this formulation though is that it does not specify how the reference class is to be selected. We should also mention Carter's anthropic principle [24], which served as an inspiration for all these ideas.
}

\section{INDEXICAL INFORMATION}

An experiment that is very easy for anyone to do is selfinspection, by which one can recognize that one exists and belongs to a certain group. The result of this experiment is usually called indexical information, and may sometimes influence the probabilities (2) [25].

To illustrate the last point, consider a special case when all observers share the same information, except for the indexical one. Suppose we have a universe populated by civilizations that can be either large, with a number of citizens $N=L$ or small, with $N=S$. These civilizations are peculiar in the following respects. First, by assumption, nobody in any of the civilizations has been able to count, or even estimate, how many individuals belong to their own civilization. Let us assume that for some reason this was hard to do. However, it suddenly becomes known that a given type of cosmological observation, which we call $\mathcal{O}$, is completely correlated with the number of people in the civilizations, so that $D_{\mathcal{O}}=N$. Second, it is also known that there is just one astronomer in each civilization, who performs the observation $\mathcal{O}$. After obtaining the result, the astronomer makes it available to the rest of the citizens in that particular civilization. Suppose everyone involved is perfectly aware of all the facts just listed, plus the additional fact that the number of small and large civilizations in the universe is the same. Given this information, the astronomers performing the observations should rightly bet that there is a $50 \%$ chance that the observation will yield $D_{\mathcal{O}}=L$ and a $50 \%$ chance that it will yield $D_{\mathcal{O}}=S$. It may therefore seem surprising that the plain citizens, who learn about the outcomes of observations by the very astronomers we just discussed, should place their bets differently.

Indeed, a plain citizen can reason as if he has been randomly picked from all the people in the multiverse, and thus he is more likely to be in a large civilization than in a small one. Hence, the plain folks should assign probabilities

$$
P\left(D_{\mathcal{O}}=L\right)=\frac{L}{L+S}, \quad P\left(D_{\mathcal{O}}=S\right)=\frac{S}{L+S} .
$$

This may seem paradoxical, since astronomers and plain folks seem to share the same information. However, this assessment is deceptive. The astronomer knows that she exists as an astronomer, whereas the citizen knows that he exists as such. Because of this difference in the indexical information their expectations for the outcome of the same experiment are, and should be, different.

Even if the astronomer, wiser than the citizens, would have to give them advice, this would have to be to use the probabilities (3). This may sound hypocritical, when the astronomer herself is using $1 / 2$ and $1 / 2$. However, after the experiment, the civilizations come to know their numbers. In small civilizations, the citizens would have done worse than the astronomer, whereas in large civilizations they 
would have turned out to do better. Hence, the astronomer minimizes liabilities by advising the citizens to predict according to (3). ${ }^{5}$

How should we then assign probabilities? Should we identify with astronomers or with regular folks? Both identifications have analogues in the definitions of probabilities that have been discussed in the literature. The probability assigned to a given environment has been defined as the probability for a randomly picked (i) civilization [4], (ii) observer [20,21], (iii) observation [22], or (iv) "observer-moment" [23] to be in that environment. The meaning of "observation" in [22] and "observer-moment" in [23] is essentially the same as the information content of an observer, so there is not much difference between (ii), (iii), and (iv). But the choice between civilizations and observers is similar to that between astronomers and regular folks in our example.

The motivation for using civilizations is that modern astronomical and particle physics measurements are collective enterprises, requiring the resources of the entire civilization. Moreover, once the measurement is made, one can assume that the result becomes available to all interested citizens. As our example shows, predictions based on the reference class of civilizations may differ from those using the reference class of individuals. In such cases, the same person may wish to bet differently as an individual and in a collective bet for the whole civilization. This would happen only in the unlikely case when the size of civilization $N$ is not known and at the same time the outcome of the measurement is correlated with $N$. When this situation does arise, the difference in predictions is not due to any defect of the theory. This difference is attributable to the different information which was used as a basis for the prediction.

\section{COMPARING DIFFERENT THEORIES}

The evidence for the theory $T$ inferred from the data set $D$ is defined as the likelihood of the data given the theory [26,27],

$$
E(T \mid D)=P(D \mid T) .
$$

If there is a number of competing theories $T_{a}$, the quantity we are interested in is the probability of a theory given the data,

\footnotetext{
${ }^{5}$ We note that indexical information can be transferred, just like all other forms of information. If, for instance, the astronomer has a "friend" whom she singles out of the crowd and with whom she communicates, then indexical information is transferred from the astronomer to that particular individual. This individual will reason that he is unlikely to have been picked by the astronomer from a large group, and will update his probabilities accordingly. As a result, his expectation for the outcome of the experiment will coincide with that of the astronomer. In our idealized example we are assuming that there is no exchange of indexical information prior to the experiment.
}

$$
P_{a} \equiv P\left(T_{a} \mid D\right)=\mathcal{N} P\left(D \mid T_{a}\right) P\left(T_{a}\right) .
$$

Here, $P\left(D \mid T_{a}\right)=E_{a}$ is the evidence for $T_{a}, P\left(T_{a}\right)$ is the prior probability for $T_{a}$, which reflects our prejudice for or against this theory, and

$$
\mathcal{N}=\left(\sum_{a} P\left(D \mid T_{a}\right) P\left(T_{a}\right)\right)^{-1}
$$

is a normalization constant. The theory having the highest probability $P_{a}$ should in principle be preferred.

The evidence $E$ is usually ranked on a logarithmic (Jeffreys) scale, and the difference in $E$ for two competing theories is deemed "significant" if $1<\Delta \ln E<2.5$, "strong" if $2.5<\Delta \ln E<5$, and "decisive" if $\Delta \ln E>$ 5 [26]. One should keep in mind though that even if the evidence in favor of one of the theories is decisive, this can be counterbalanced by a strong enough bias against that theory in the prior.

\section{Humans vs Jovians}

Hartle and Srednicki [7] recently discussed an example intended to show that the assumption of typicality in a wider reference class can lead to absurd conclusions. This is an example where indexical information plays an important role. Suppose we have a theory $T_{1}$ predicting that there is no life on Jupiter and theory $T_{2}$ that says that Jupiter is populated by intelligent beings and has population size $J$ much greater than the human population $H$ on Earth, $J \gg H$. According to $T_{1}$, we are typical observers in the Solar System, and according to $T_{2}$ we are not. Hartle and Srednicki argue that it would be absurd to dismiss $T_{2}$ merely because it makes us atypical. We note, however, that the principle of mediocrity does not necessarily imply that $T_{2}$ should be disfavored. This is because a smaller evidence can be compensated for by a higher prior in Eq. (5).

The prior simply reflects our preference for the theory before we perform the measurement, and to our knowledge there is no generally agreed upon method for calculating it. For instance, one may adopt the so-called self-indication assumption (SIA), which asserts that we should, all other things being equal, assign a higher probability to the theory predicting a larger number of observers. SIA was first introduced by Dieks [28] and further discussed by Bostrom [23]. Olum [29] presented persuasive arguments that SIA should be adopted, but it still remains somewhat controversial. ${ }^{6}$ The question of validity of the SIA is peripheral to the issues we are concerned with here. For the sake of the argument, in this section we shall adopt the SIA.

Suppose some individual (let us call him $K$ ) inspected himself enough to tell that he is an intelligent observer in

\footnotetext{
${ }^{6}$ One of the difficulties of the SIA is the so-called "presumptuous philosopher" problem, as discussed in [23].
} 
the Solar System, but he does not know yet whether he is human or Jovian. Then the principle of mediocrity mandates that he regard himself as randomly picked from the reference class $C_{H J}$ including all human and Jovian observers in the Solar System. According to $T_{1}$ this class coincides with the class $C_{H}$ of humans and has $H$ individuals, and according to $T_{2}$ it is much wider than $C_{H}$ and has $(J+H)$ individuals. The probability for $K$ to exist at all is proportional to the total number of individuals. Thus, having obtained the indexical information that he exists, he should update the prior probabilities for the two theories by enhancing the probability for $T_{2}$ by the factor $(J+H) / H$ relative to that for $T_{1}$.

Before taking into account any indexical information, we may characterize the two theories by prior probabilities $P\left(T_{1}\right)$ and $P\left(T_{2}\right)$. Then, according to the SIA, the fact of the observer's existence (which we will also denote by $K$ ) updates such probabilities to $P\left(T_{1} \mid K\right)=P\left(T_{1}\right) H /(2 H+$ $J)$, and $P\left(T_{2} \mid K\right)=P\left(T_{2}\right)(H+J) /(2 H+J)$.

Suppose now that $K$ looks out of the window and discovers that he is on Earth. According to $T_{2}$ this second piece of indexical information has probability $P(K \in$ $\left.H \mid T_{2}\right)=H /(J+H)$, and the updated probability for $T_{2}$ is

$$
\begin{aligned}
P_{2} & =P\left(T_{2} \mid K ; K \in H\right) \\
& =P\left(T_{2} \mid K\right) P\left(K \in H \mid T_{2}\right) / P(K \in H),
\end{aligned}
$$

where $\quad P(K \in H)=P\left(K \in H \mid T_{1}\right) P\left(T_{1} \mid K\right)+P(K \in$ $\left.H \mid T_{2}\right) P\left(T_{2} \mid K\right)$ is the total probability for $K$ to be human (after the first piece of indexical information-that $K$ exists - has been used). On the other hand, the observation that $K$ is human is guaranteed in the theory $T_{1}, P(K \in$ $\left.H \mid T_{1}\right)=1$ and therefore

$$
P_{1}=P\left(T_{1} \mid K ; K \in H\right)=P\left(T_{1} \mid K\right) / P(K \in H) .
$$

The net result is that $P_{2} / P_{1}=P\left(T_{2}\right) / P\left(T_{1}\right)$. In other words, the observation that $K$ is human does not give him any information about the existence of life on Jupiter.

In conclusion, the principle of mediocrity does not necessarily make $T_{2}$ less likely than $T_{1}$ in spite of the fact that we are less typical in $T_{2}$ than in $T_{1}$. In the example above we have avoided this conclusion by using the selfindication assumption (SIA). We reiterate that this assumption is somewhat controversial, as is the more general problem of assigning prior probabilities to different theories. This is nevertheless an interesting subject which we leave for future discussion.

\section{PREDICTION VS EXPLANATION}

Predicting the results of future measurements may be too narrow a goal for a theory of the multiverse. Many constants of nature and cosmological parameters have already been measured, so we have missed the opportunity to predict them, but we can still try to explain their values.
In order to explain the observed value of some parameter $\alpha$, we can utilize the same approach as we outlined above, except we now have to ignore the information we have about $\alpha$. In other words, we should consider a broader reference class $C^{(\alpha)}$, including observers who measured the same values of the constants as we did, except for the value of $\alpha$, which is allowed to vary from one observer to another. This reference class is then divided into subclasses, $C_{j}^{(\alpha)}$, according to the measured values $\alpha_{j}$, and the corresponding branching ratios are interpreted as probabilities $P\left(\alpha_{j}\right)$. The evidence for the underlying theory can be evaluated using Eq. (4), as before. This evidence can be used to discriminate between different models. In the same manner, one can introduce still broader reference classes, $C^{(\alpha, \beta, \ldots)}$, by allowing several parameters to vary.

As an illustration, we consider the case of the cosmological constant $\Lambda$. (For a review, see [30]. More recent discussion can be found in [31-33].) In order to explain the smallness of the observed value $\Lambda_{*}$, we choose a reference class $C^{(\Lambda)}$ which includes all humanlike observers performing independent measurements of $\Lambda$ in parts of the universe where all particle physics and cosmological parameters other than $\Lambda$ have the same values as in our local region. The corresponding distribution can be represented as

$$
P_{1}(\Lambda) \propto P_{V}(\Lambda) n_{\mathrm{obs}}(\Lambda),
$$

where the volume distribution $P_{V}(\Lambda)$ characterizes the fraction of (comoving) volume occupied by regions with a given value of $\Lambda$ and $n_{\text {obs }}(\Lambda)$ is the number density of observers belonging to the chosen reference class.

The standard argument suggests $[5,34,35]$ that the volume distribution is well approximated by

$$
P_{V}(\Lambda) \approx \text { const, }
$$

because the anthropic range where $n_{\mathrm{obs}}(\Lambda)$ is appreciably different from zero is much narrower than the full range of $\Lambda$. Note that a logarithmic distribution, which gives equal likelihood to all orders of magnitude (the so-called Jeffreys prior) would not be a suitable choice for $P_{V}(\Lambda)$. We know that Lambda is a sum of very large positive and negative contributions which are fine-tuned to near cancellation. Decreasing the order of magnitude of $\Lambda$ corresponds to a higher level of fine-tuning and should be given a lower probability. The "standard argument" that we referred to in the text assumes that $\Lambda=0$ is not a special point of the volume distribution. (This point is singular for the Jeffreys prior.) Perhaps more importantly, the volume distribution $P_{V}(\Lambda)$ is not a prior in the usual sense. It is a calculable distribution and has been demonstrated to be flat in some multiverse models [36-39].

For the anthropic factor $n_{\mathrm{obs}}(\Lambda)$, the analysis of positive and negative $\Lambda$ is rather different; for our purposes it will be sufficient to focus on $\Lambda>0$. Then the density of observers is usually approximated as 


$$
n_{\mathrm{obs}} \propto F\left(M>M_{G}, \Lambda\right),
$$

where the quantity on the right-hand side is the asymptotic fraction of baryonic matter which clusters into objects of mass greater than the characteristic galactic mass $M_{G}$. The idea here is that there is a certain average number of stars per unit baryonic mass and certain number of observers per star, and that these numbers are not strongly affected by the value of $\Lambda$ in the range of interest. Using the PressSchechter formalism to approximate the fraction of collapsed matter $F$ and combining Eqs. (7)-(9), one finds $[6,40]$

$$
P_{1}(\Lambda)=\frac{\sqrt{\pi}}{\Lambda_{1}} \operatorname{erfc}\left[\left(\Lambda / \Lambda_{1}\right)^{1 / 3}\right]
$$

Here,

$$
\Lambda_{1} \approx 2 \rho_{m} \sigma_{G}^{3},
$$

$\rho_{m}$ is the density of nonrelativistic matter and $\sigma_{G}$ is the linearized density contrast on the galactic scale. The prefactor in (10) is chosen so that the distribution $P_{1}(\Lambda)$ is properly normalized. With the observed values of $\rho_{m}$ and $\sigma_{G}$, the quantity $\Lambda_{1}$ in (11) is comparable to the observed value $\Lambda_{*}, \Lambda_{1} \approx 3 \Lambda_{*}$.

An alternative explanation for the observed value of $\Lambda$ is that $\Lambda$ just is what it is. It has to have some value and we have found it. There has been no anthropic selection. In this approach, $\Lambda$ has no reason to be smaller than the value set by the supersymmetry breaking scale, $\Lambda_{\text {SUSY }}$. No particular value below that scale is preferred, so the prior distribution for $\Lambda$ can be set as $P_{2}(\Lambda)=\Lambda_{\text {SUSY }}^{-1}$ for $\Lambda<\Lambda_{\text {SUSY }}$ and $P_{2}(\Lambda)=0$ for $\Lambda>\Lambda_{\text {SUSY }}$. Assuming that the measurement errors are small, $\delta \Lambda \ll \Lambda_{*}$, we have

$$
\begin{aligned}
E_{1} / E_{2} & =P_{1}\left(\Lambda_{*}\right) / P_{2}\left(\Lambda_{*}\right)=\sqrt{\pi} \frac{\Lambda_{\mathrm{SUSY}}}{\Lambda_{1}} \operatorname{erfc}\left[\left(\Lambda_{*} / \Lambda_{1}\right)^{1 / 3}\right] \\
& \approx 0.2 \frac{\Lambda_{\mathrm{SUSY}}}{\Lambda_{*}} .
\end{aligned}
$$

We know that $\Lambda_{\text {SUSY }} \gtrsim(1 \mathrm{TeV})^{4}$, and thus $E_{1} / E_{2} \gtrsim 10^{60}$. Comparison of the two theories on the Jeffreys scale gives $\Delta \ln E>138$, and thus the evidence in favor of the multiverse model is decisive.
If one is not interested in explanation and wants to stick to the business of prediction, the calculation should be somewhat different. Imagine we are back in the mid1990 's, when the value of $\Lambda$ was not known, and we want to make a prediction for the upcoming supernova measurements. The current upper bound on $\Lambda$ at the time was [41]

$$
\Lambda \lesssim 4 \rho_{m}
$$

The values of some relevant parameters, such as $\sigma_{G}$ were known with a rather poor accuracy, but to simplify the discussion we shall assume as before that all parameters other than $\Lambda$ have been accurately measured.

The quantity $4 \rho_{m}$ is somewhat less than $\Lambda_{1}$ in (11); hence the distribution (10) in the range (13) can be roughly approximated as flat,

$$
P(\Lambda) \approx \frac{1}{4 \rho_{m}}=\text { const. }
$$

The probability for $\Lambda$ to be smaller than any given value is then $P(<\Lambda) \approx \Lambda / 4 \rho_{m}$. For example, $P\left(<0.2 \rho_{m}\right) \approx 0.05$. Thus, in this simplified model, one can predict at $95 \%$ confidence level that $\Lambda>0.2 \rho_{m}$. This is the essence of the prediction made in Refs. [3-6]. We note that prediction and explanation were not cleanly separated in Refs. [3-6]. Rather, they gave a mixture of both. ${ }^{7}$

\section{ACKNOWLEDGMENTS}

We would like to thank Alan Guth, Andrew Liddle, Ken Olum, and Michael Salem for very useful discussions. We are also grateful to Misao Sasaki and the Yukawa Institute for their hospitality at the workshop YITP-W-07-10, where this work was originated. This work was supported in part by the Fundamental Questions Institute (J. G. and A. V.), by grants No. FPA2007-66665C02-02 and No. DURSI 2005SGR-00082 (J.G.), and by the National Science Foundation (A. V.).

\footnotetext{
${ }^{7}$ Anthropic explanations for the smallness of $\Lambda$ have been suggested earlier in $[42,43]$.
}

[1] See, e.g., L. Susskind, in Universe or Multiverse, edited by B. J. Carr (Cambridge University Press, Cambridge, England, 2006).

[2] For recent reviews of eternal inflation, see A.H. Guth, J. Phys. A 40, 6811 (2007); S. Winitzki, arXiv:hep-th/ 0706.3994.

[3] S. Weinberg, Rev. Mod. Phys. 61, 1 (1989).

[4] A. Vilenkin, Phys. Rev. Lett. 74, 846 (1995).
[5] G. Efstathiou, Mon. Not. R. Astron. Soc. 274, L73 (1995).

[6] H. Martel, P. R. Shapiro, and S. Weinberg, Astrophys. J. 492, 29 (1998).

[7] J. B. Hartle and M. Srednicki, Phys. Rev. D 75, 123523 (2007).

[8] G. D. Starkman and R. Trotta, Phys. Rev. Lett. 97, 201301 (2006). 
[9] I. Maor, L. Krauss, and G. Starkman, arXiv:hep-th/ 0709.0502 [Phys. Rev. Lett. (to be published)].

[10] J. Garriga, D. Schwartz-Perlov, A. Vilenkin, and S. Winitzki, J. Cosmol. Astropart. Phys. 01 (2006) 017.

[11] A. Linde, J. Cosmol. Astropart. Phys. 06 (2007) 017.

[12] M. Tegmark, J. Cosmol. Astropart. Phys. 04 (2005) 001.

[13] R. Bousso, R. Harnik, G. D. Kribs, and G. Perez, Phys. Rev. D 76, 043513 (2007).

[14] J. M. Cline, A. R. Frey, and G. Holder, arXiv:0709.4443.

[15] For a review see A. Vilenkin, J. Phys. A 40, 6777 (2007).

[16] J. Garriga and A. Vilenkin, Phys. Rev. D 64, 043511 (2001).

[17] R. M. Neal, arXiv:math/0608592.

[18] L. Pogosian, M. Tegmark, and A. Vilenkin, J. Cosmol. Astropart. Phys. 07 (2004) 005; M. Tegmark, A. Vilenkin, and L. Pogosian, Phys. Rev. D 71, 103523 (2005).

[19] D. N. Page, arXiv:hep-th/0707.4169.

[20] J. R. Gott, Nature (London) 363, 315 (1993).

[21] J. Leslie, Universes (Routledge, New York, 1989).

[22] D. N. Page, Int. J. Mod. Phys. D 5, 583 (1996).

[23] N. Bostrom, Anthropic Bias: Observational Selection Effects in Science and Philosopny (Routledge, New York, 2002).

[24] B. Carter, in Confrontation of Cosmological Theories with the Data, edited by M.S. Longair (Reidel, Dordrecht, 1974).

[25] N. Bostrom, Erkenntnis 52, 93 (2000).

[26] H. Jeffreys, Theory of Probability (Clarendon Press, Oxford, 1939).

[27] For application of Bayesian evidence to cosmological model selection see, e.g., A. R. Liddle, P. Mukherjee, and D. Parkinson, A\&G 47, 4.30 (2006).
[28] D. Dieks, Phil. Q. 42, 78 (1992).

[29] K. Olum, Phil. Q. 52, 164 (2002).

[30] A. Vilenkin, in Universe or Multiverse, edited by B. J. Carr (Cambridge University Press, Cambridge, England, 2006).

[31] M. Tegmark, A. Aguirre, M. J. Rees, and F. Wilczek, Phys. Rev. D 73, 023505 (2006).

[32] L. Pogosian and A. Vilenkin, J. Cosmol. Astropart. Phys. 01 (2007) 025.

[33] J. A. Peacock, Mon. Not. R. Astron. Soc. 379, 1067 (2007).

[34] A. Vilenkin, in Cosmological Constant and the Evolution of the Universe, edited by K. Sato, T. Suginohara, and N. Sugiyama (Universal Academy Press, Tokyo, 1996).

[35] S. Weinberg, in Critical Dialogues in Cosmology, edited by N. G. Turok (World Scientific, Singapore, 1997).

[36] J. Garriga and A. Vilenkin, Phys. Rev. D 61, 083502 (2000); 64, 023517 (2001); 67, 043503 (2003).

[37] S. Weinberg, Phys. Rev. D 61, 103505 (2000).

[38] K. D. Olum and D. Schwartz-Perlov, J. Cosmol. Astropart. Phys. 10 (2007) 010.

[39] T. Clifton, S. Shenker, and N. Sivanandam, J. High Energy Phys. 09 (2007) 034.

[40] J. Garriga, M. Livio, and A. Vilenkin, Phys. Rev. D 61, 023503 (1999).

[41] M. Fukugita, in Critical Dialogues in Cosmology, edited by N. Turok (Princeton University, Princeton, NJ, 1997).

[42] S. Weinberg, Phys. Rev. Lett. 59, 2607 (1987).

[43] A. D. Linde, in 300 Years of Gravitation, edited by S. W. Hawking and W. Israel (Cambridge University Press, Cambridge, England, 1987). 